\title{
SYSTOLIC SPARSE MATRIX VECTOR MULTIPLY IN THE AGE OF TPUS AND ACCELERATORS
}

\author{
Euripides Montagne \\ Department of Computer Science \\ University of Central Florida \\ 4000 Central Florida Boulevard \\ Orlando, FL, USA \\ eurip@ucf.edu
}

\author{
Rina Surós \\ Faculty of Science \\ University Central de Venezuela \\ Av. Paseo los Illustres \\ Caracas, DC, VENEZUELA \\ rsuros@gmail.com
}

\begin{abstract}
Tensor Processing Units has brought back systolic arrays as a computational alternative to high performance computing. Recently Google presented a Tensor Processing Unit for handling matrix multiplication using systolic arrays. This unit is designed for dense matrices only. As they stated, sparse architectural support was omitted momentarily but they will focus on sparsity in future designs. We propose a systolic array to compute the Sparse Matrix Vector product in $T 2(n) \approx\left\lceil\frac{n n z}{2}\right\rceil+2 n+2$ using $2 n+2$ processing elements. The systolic array we propose also use accumulators to collect the partial results of the resulting vector and supports adapting tiling.
\end{abstract}

Keywords: systolic arrays, sparse matrix, spmv product, tensor processing unit(tpu).

\section{INTRODUCTION}

Since Tensor Processing Units (TPUs) were announced by Google there has been a resurgence of systolic arrays as application accelerators in this called, by some people, a new golden age of computer science (Jouppi et al. 2017). Systolic arrays were proposed by Kung and Leiserson in 1978 as an accelerator to speed up execution of applications related to matrix computation (Kung and Leiserson 1979). These accelerators allow the execution of multiples computations for each memory access without increasing I/O requirements (Kung 1982). A systolic array consists of a network of tiny Processing Elements (PE) where each data item that enters the network moves synchronously from one PE to another allowing multiples data items to coexist in different PEs. This coexistence creates a natural concurrent environment where each PE acts on a different data item simultaneously making thus systolic arrays a highly parallel architecture suitable to solve specific problems. Initially one can think of a systolic array as a network of PEs all executing the same computation, say an inner product, however this is not always the case. We can extent this conception by creating systolic arrays which combine two or three different types of PEs (Kung 1982).

The sparse matrix vector product is a computational kernel used in many applications including neural networks and deep learning. As a never ending story, many researchers since the early computers were conceived have had interest in running the SpMV kernel faster and faster. Many mappings based in CSR and partitioning have been studied and implemented in multiprocessors, multicores, GPUs, and FPGA accelerators (Im et al. 2004, Krotkiewski and Dabrowski 2010, Vuduc 2004, Williams et al. 2009). CSR is widely known as the facto standard due to its effectiveness and efficiency to deal with sparse matrices 
and perhaps this is the reason CSR is frequently used to map the SpMV product in all types of architectures. However less popular methods as CSC are giving apparently better results on FPGA and Application Specific-Integrated Circuits (ASIC) accelerators (Zhuo and Prasanna 2005, Dorrance et al. 2014, Han et al. 2016) because the algorithms using CSC does not access array $x$ randomly as the algorithms based on CSR do and it seems this is an advantage when a lower level implementation is carried out.

In this work we propose a systolic array with $2 n+2$ processing elements. There are two types of PE: two multipliers and $2 n$ selective adders. Inspired by the architecture of TPU, we also use accumulators to store the partial computations of each element of the resulting vector. This systolic array computes the SpMV in $T 2(n) \approx\left\lceil\frac{n n z}{2}\right\rceil+2 n+2$, where $n n z$ stands for number of non-zeros. The matrices used in this study were selected from Matrix Market database (Boisvert, Pozo, Remington, Barrett, and Dongarra 1997) and The University of Florida Sparse Matrix Collection (Davis and Hu 2011).

\section{SPARSE MATRIX COMPRESSION WITH TJDS}

Performance optimizations of the SpMV multiplication using compression formats has been the focus of many research projects since the times programs were executed solely sequentially. Several formatting options have been reported to compress sparse matrices to gather the non zero values into a data structure that allows to run the SpMV product computation efficiently without handling the zero values. We present the Transpose Jagged Diagonal Storage (TJDS) compression format as an alternative for mapping the SpMV multiplication onto a systolic array (Montagne and Ekambaran 2004). The SpMV multiplication, $y=A x$, of a sparse matrix $A$ and dense vector $x$, is expressed in matrix form as follows:

$$
\left[\begin{array}{l}
y_{1} \\
y_{2} \\
y_{3} \\
y_{4} \\
y_{5}
\end{array}\right]=\left[\begin{array}{ccccc}
a_{11} & a_{12} & 0 & a_{14} & 0 \\
0 & a_{22} & 0 & a_{24} & 0 \\
0 & 0 & a_{33} & 0 & 0 \\
0 & 0 & a_{43} & a_{44} & 0 \\
0 & 0 & a_{53} & 0 & a_{55}
\end{array}\right]\left[\begin{array}{l}
x_{1} \\
x_{2} \\
x_{3} \\
x_{4} \\
x_{5}
\end{array}\right]
$$

To explain how to compress a sparse matrix $A$ using the transpose jagged diagonal format step by step, we will used two data structures named, $A_{d s}$ containing the matrix coefficients and $I_{d s}$ containing the row indices of the matrix coefficients in the original matrix as shown below:

$$
\begin{gathered}
A_{d s}=\left|\begin{array}{ccccc}
a_{11} & a_{12} & 0 & a_{14} & 0 \\
0 & a_{22} & 0 & a_{24} & 0 \\
0 & 0 & a_{33} & 0 & 0 \\
0 & 0 & a_{43} & a_{44} & 0 \\
0 & 0 & a_{53} & 0 & a_{55}
\end{array}\right| \\
I_{d s}=\left|\begin{array}{ccccc}
1 & 1 & 0 & 1 & 0 \\
0 & 2 & 0 & 2 & 0 \\
0 & 0 & 3 & 0 & 0 \\
0 & 0 & 4 & 4 & 0 \\
0 & 0 & 5 & 0 & 5
\end{array}\right|
\end{gathered}
$$

Then non-zero coefficients in the data structures $A_{d s}$ and the row indexes $I_{d s}$ are compressed column-wise and reordered in decreasing order of the number of non-zero elements per column. The elements of the vector $x$ are reordered accordingly as if they were an additional row of matrix $A$ and vector $y$ remains the same. This arrangement is illustrated as follows: 


$$
\begin{gathered}
A_{d s}=\left|\begin{array}{ccccc}
a_{33} & a_{14} & a_{12} & a_{11} & a_{55} \\
a_{43} & a_{24} & a_{22} & 0 & 0 \\
a_{53} & a_{44} & 0 & 0 & 0 \\
0 & 0 & 0 & 0 & 0 \\
0 & 0 & 0 & 0 & 0
\end{array}\right| \\
I_{d s}=\left|\begin{array}{ccccc}
3 & 1 & 1 & 1 & 5 \\
4 & 2 & 2 & 0 & 0 \\
5 & 4 & 0 & 0 & 0 \\
0 & 0 & 0 & 0 & 0 \\
0 & 0 & 0 & 0 & 0
\end{array}\right|\left[\begin{array}{l}
x_{3} \\
x_{4} \\
x_{2} \\
x_{1} \\
x_{5}
\end{array}\right]\left[\begin{array}{l}
y_{1} \\
y_{2} \\
y_{3} \\
y_{4} \\
y_{5}
\end{array}\right]
\end{gathered}
$$

The final step consists in mapping the above rearranged coefficients and indices into a set of linear arrays. To do so, we store each row or tile of the nonzero coefficients of $A_{d s}$ in a linear array called val one row after another. Each one of these rows is called a transpose jagged diagonal $(t j d)$ or $t j \_t i l e$. In our example the number of $t j d$ (num_t $j d$ ) is three. Similarly, all rows of $I_{d s}$ are stored one after the other in another linear array denoted as row_index. Finally, a third array called start of lenth num_t $j d+1$ stores the starting position of each $t j \_t i l e$ stored in array val. Notice that the number of $t j_{-}$tiles is equal to the numbers of

\begin{tabular}{|c|c|c|c|c|c|c|c|c|c|c|c|c|}
\hline val & $a_{33}$ & $a_{14}$ & $a_{12}$ & $a_{1}$ & & 155 & $a_{43}$ & $a_{24}$ & $a_{22}$ & $a_{5}$ & & $a_{44}$ \\
\hline & \multicolumn{3}{|c|}{ row_index } & \begin{tabular}{l|l}
3 & 1
\end{tabular} & 1 & 1 & 5 & \begin{tabular}{l|l}
4 & 2
\end{tabular} & \begin{tabular}{l|l}
2 & 5
\end{tabular} & 4 & & \\
\hline & & & & tart & 1 & 6 & 9 & 11 & & & & \\
\hline & & & $X$ & $x_{3}$ & $x_{4}$ & $x_{2}$ & $x_{1}$ & $x_{5}$ & & & & \\
\hline & & & $Y$ & $y_{1}$ & $y_{2}$ & $y_{3}$ & $y_{4}$ & $y_{5}$ & & & & \\
\hline
\end{tabular}
coefficients in the most populated column. These arrays are shown below:

TJDS is a scatter base compressed storage format which produces long vectors and this makes it suitable for using on systolic arrays. We can characterize it as follows:

- The matrix coefficients in array val are accessed sequentially.

- The elements of the source vector $\mathrm{x}$ are accessed sequentially and are aligned elementwise with the coefficients of $A$ stored in vector val.

- The number of tiles is smaller than $n$ (num_tjd $<<n$ ).

- Source array $\mathrm{x}$ have to be permuted before the computation.

It is worth mentioning that CSR produces $n$ tiles while TJDS generates num_ $t j d$. To illustrate this comparison, let us take as an example matrix soc-sign-bitcoin-otc which is a $5881 \times 5881$ sparse matrix with 35,592 $n n z$ coefficients. This matrix generates 535 tiles using TJDS and 5,881 tiles with CSR. 


\section{A SYSTOLIC ARRAY FOR THE SPARSE MATRIX VECTOR PRODUCT}

Let us describe, for this example, how these data structures are used to perform the matrix-vector product $y=A x$ using a systolic array.

\begin{tabular}{|c|c|c|c|c|c|c|c|c|c|c|c|c|}
\hline val & $a_{33}$ & $a_{14}$ & $a_{12}$ & $a_{1}$ & \multicolumn{2}{|c|}{$a_{55}$} & $a_{43}$ & $a_{24}$ & $a_{22}$ & \multicolumn{2}{|c|}{$a_{53}$} & $a_{44}$ \\
\hline & \multicolumn{3}{|c|}{ row_index } & \begin{tabular}{l|l}
3 & 1
\end{tabular} & 1 & 1 & 5 & \begin{tabular}{l|l}
4 & 2
\end{tabular} & 2 & 5 & 4 & \\
\hline & & & & $\operatorname{tart}$ & 1 & 6 & 9 & 11 & & & & \\
\hline & & & $\mathrm{X}$ & $x_{3}$ & $x_{4}$ & $x_{2}$ & $x_{1}$ & $x_{5}$ & & & & \\
\hline & & & $Y$ & $y_{1}$ & $y_{2}$ & $y_{3}$ & $y_{4}$ & $y_{5}$ & & & & \\
\hline
\end{tabular}

There are three transpose jagged diagonal or row_tiles $\left(t j \_\right.$diag) in array VAL and array start will allow us to determine the size of each $t j \_$diag using the following expression

$$
\operatorname{start}[i+1]-\operatorname{start}[i] \quad \text { with } \quad i=1,2,3 .
$$

Taken a closer look to the first $t j \_$diag in VAL and vector $\mathrm{X}$ you will notice that we can carry out the operation $a_{i, j} \times x_{j}$ elementwise because TJDS provides the alignment of the two vectors. Each element in array row_index gives us information about where to accumulate and store each partial product for each element of the resulting array $Y$.

To compute the SPMV using systolic arrays, we will explain the way the sparse matrix vector product is computed using the basic mechanism which consists of TPU-like linear systolic array with $n+1$ Processing Elements (PE). This arrangement is depicted in Figure 1. There are two type of processing elements a multiplier (round shape) and $n$ selective adders (rectangular shape). We call it TPU-like because outside the systolic network there are accumulators to capture partial inner products to compute the resulting vector $Y$. Figure 2 shows a closer look of the systolic array processing elements and accumulators.



Figure 1: Systolic Array for the Sparse Matrix Vector Multiply.

As follows we provide a detailed description of the actions taken by each PE and the accumulators:

- In this arrangement, the systolic array left-most PE (round one) reads in a matrix coefficient $a_{i j}$ from array VAL and an element $x_{j}$ from array $\mathrm{X}$ and computes $a_{i j} \times x_{j}$. Then it pumps out to the right the product $a_{i j} x_{j}$.

- All rectangular Processing Elements get a $a_{i j} \times x_{j}$, an index $i$, and a $y_{k}$. The $a_{i j} \times x_{j}$ and index $i$ are read in from the neighbor PE on its left except for the one neighbouring the multiplier PE which 




Figure 2: Closer look to the PEs of the systolic array for the SpMV.

reads in index $i$ from row_index array. The $y_{k}$, with initial value zero, enters the PE from the top and the subscript $k$ identifies the element of the resulting vector. Then the following actions are carried out.

(i) Index $i$ is decremented by one.

(ii) If $(i==0)$ computes $y_{i}=y_{i}+a_{i j} \times x_{j}$.

(iii) Pumps out downwards $y_{k}$ and pumps out to the left both $a_{i j} x_{j}$ and $i$.

- Each Accumulator collects all partial result of a specific $y_{k}$ element and add them up to provide the resulting $y$ vector.

Now let us see how the computation is carried out step by step. Figures 3 shows the initial stage for the computation of the SpMV. The elements of the first tile of the Val array and the elements of array $x$ are at the input of the multiplier (round PE). The elements of row_index array are at the input of the left-most rectangular PE and will enter the PE delayed by one time unit. You can observe the resulting array $y$, initially zero, enter the selective adders from the top. Each $y_{k}=0$ with $(k=1,2,3, \ldots, n)$ enters a different selective adder PE at each step.

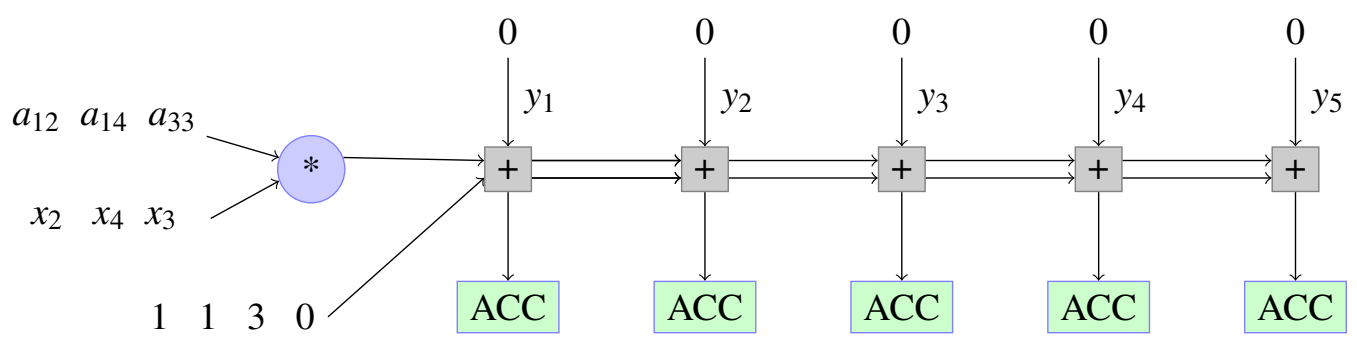

Figure 3: Initial stage to compute the SpMV on a sytolic array.

Figures 4 illustrate the first computational step. In this step data begin to flow into the systolic array and only the round PE executes useful computation. Then Figure 5 illustrate two simultaneous operations: One carried out by the round PE and another one by the selctive adder associated to $y_{1}$. The next computacional step shows three simultaneous operations: one by the round PE, one by the selective adder associated to 
$y_{1}$ and another one carried out by the selective adder associated to $y_{2}$. Figure 6 presents this step and give details about the computations executed by each PE. You can observe in this Figure that the value $a_{14} x_{4}$ is about to enter the accumulator associated with $y_{1}$. Figure 7 Shows four PEs acting simultaneously. Partial results are sent down to accumulators associated with $y_{1}$ and $y_{3}$. The accumulator associated with $y_{2}$ receives a zero.

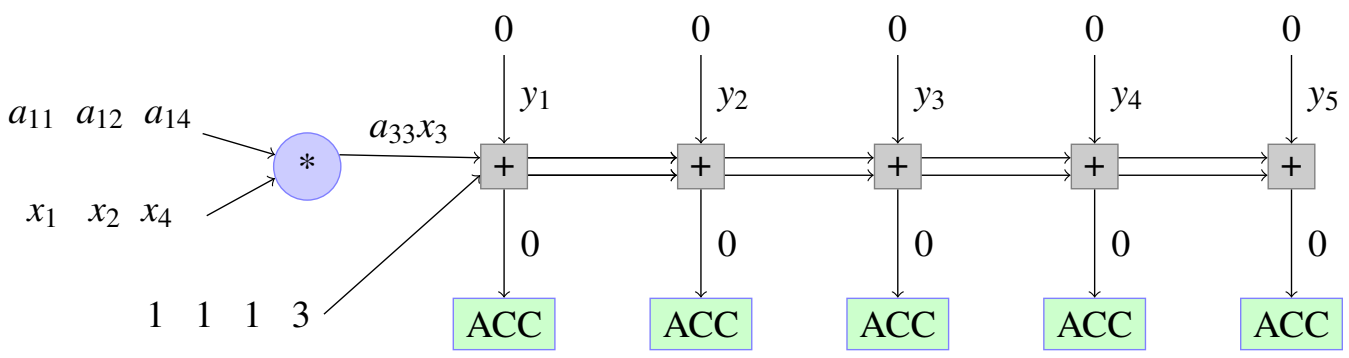

Figure 4: (Step 1) Round PE reads in $a_{33}$ and $x_{3}$, computes $a_{33} \times x_{3}$ and pumps it out to the right.

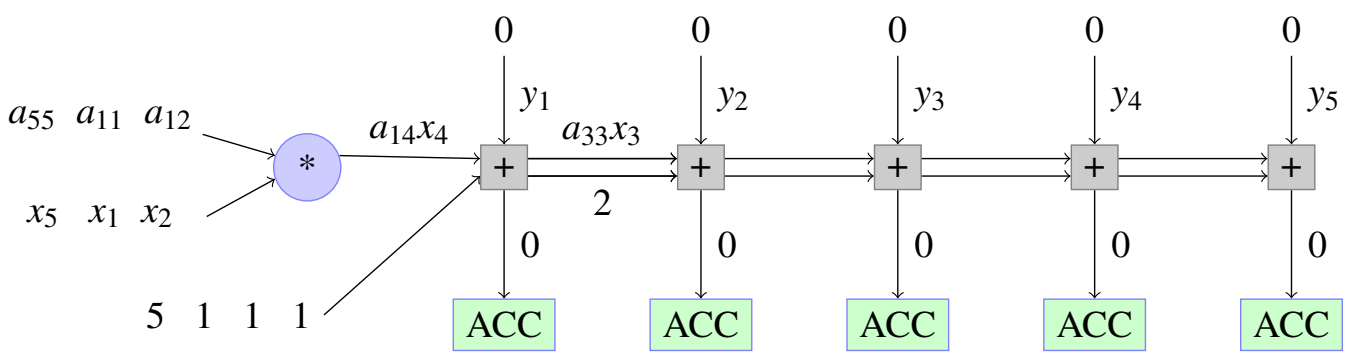

Figure 5: (Step 2) Round PE reads in $a_{14}$ and $x_{4}$, computes $a_{14} \times x_{4}$ and pumps it out to the right. Simultaneously, the rectangular PE associated to $y_{1}$ reads in $y_{1}=0, a_{33} \times x_{3}$ and row index $i$ from vector rindex. Decrements $i$ by one and if $i==0$ computes $y_{3}=y_{3}+a_{33} \times x_{3}$. As $i \neq 0$, the $y_{1}$ is just forwarded downwards while $a_{33} \times x_{3}$, and new $i$ value are pump out to the left.

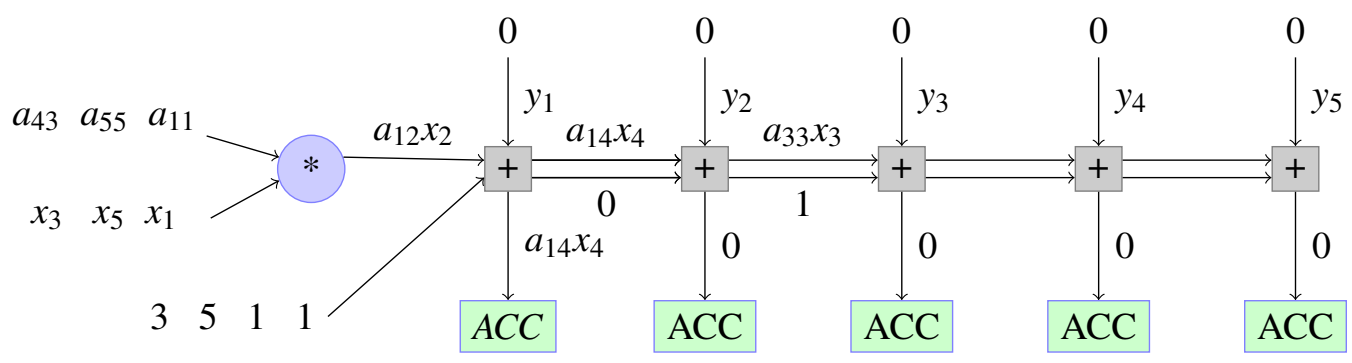

Figure 6: (Step 3) Round PE reads in $a_{12}$ and $x_{2}$, computes $a_{12} \times x_{2}$ and pumps it out to the right. Simultaneously, the leftmost rectangular PE reads in $a_{14} \times x_{4}$ and row index $i$ from vector rindex. Decrements $i$ by one and if $i==0$ computes $y_{1}=y_{1}+a_{14} \times x_{4}$. In parallel the adder associated $y_{2}$ reads in $a_{33} \times x_{3}$ and index $i$; If $i==0$ the adder computes $y_{3}=y_{3}+a_{33} \times x_{3}$, then $a_{33} \times x_{3}$ and the new $i$ value are pumped out to the left and $y_{2}$ downwards.

Now that the basic mechanism is understood, we propose a systolic array with $2 n+2$ Processing elements (PE). This SA consists of a duplication of the linear array introduced as the basic mechanism. Figure 8 illustrates this arrangement. This new arrangement will speed up the computation of the SpMV because we can split the input values and run half of the computation on each array or a different tile on each linear 
array. In our example, the best performance will be obtained by triplicating the basic mechanism because each array will handle a different tile in parallel but the cost in resources needed would be excessive.

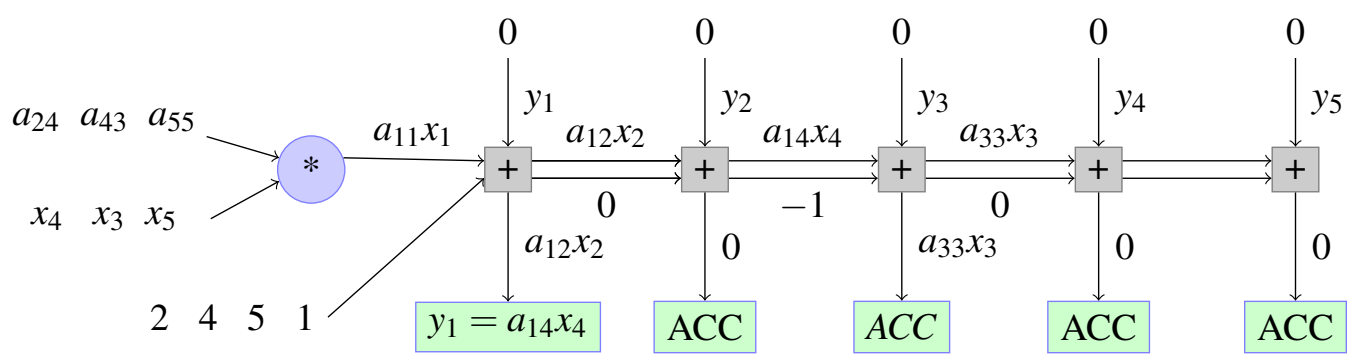

Figure 7: (Step 4) In this step the multiplier and three selective adders are active. Adders associated to $y_{1}$ and $y_{3}$ produced partial results and sent them to accumulators while the adder associated to $y_{2}$ forwards a zero to its accumulator. The multiplier pumps out a new partial result.

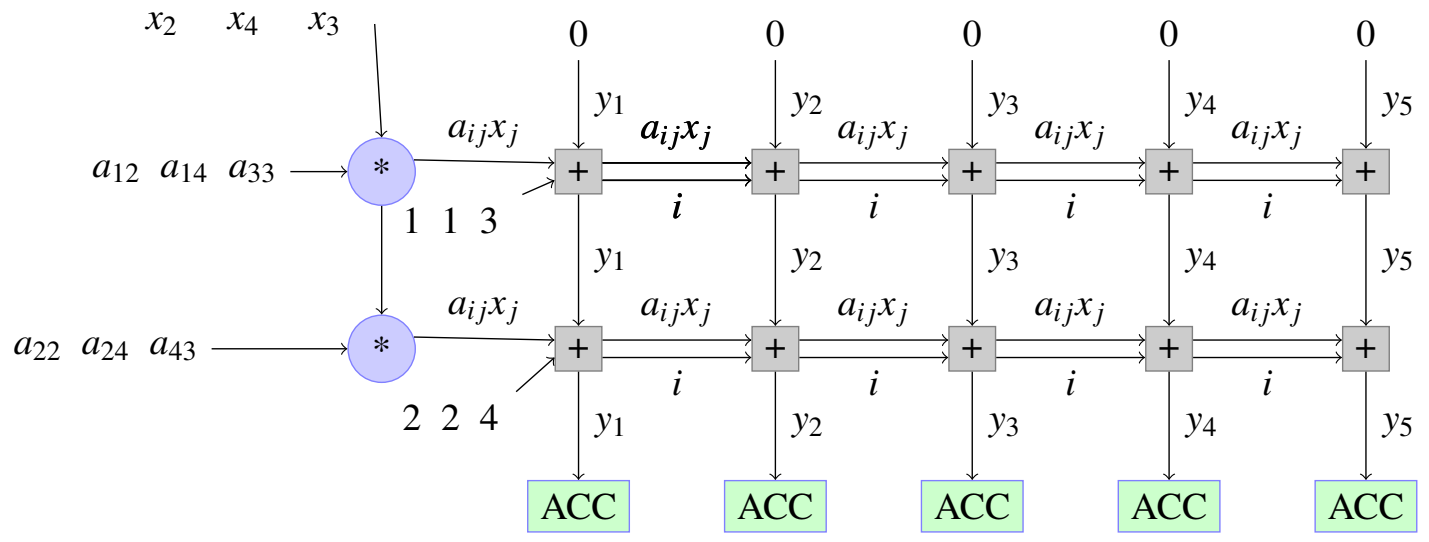

Figure 8: Systolic Array for the Sparse Matrix Vector Multiply.

\section{TIME ANALYSIS}

We will present the time analysis to compute the SpMV on the linear systolic array denoted as the basic mechanism. In this systolic array there are $n+1 \mathrm{PE}$ and all the $n n z$ coefficients of $A$ must traverse the systolic network. There is no knowledge of what element of the resulting vector $y$ will be updated by the last coefficient that enters the systolic array. Therefore we must consider the possibility of updating $y_{n}$. Furthermore, an additional step must be considered to update a $y_{i}$ element by the accumulator. Finally $n$ steps are required to pump out the resulting vector $y$.

The time to compute the SpMV using the basic mechanism consisting of a linear array is presented as T1(n) in Table 1 and is given by the upper bound:

$$
n n z+n+1 \leq T 1(n) \leq n n z+2 n+1 .
$$

By making $k=\left\lceil\frac{n n z}{n}\right\rceil$, we can rewrite the equation as:

$$
(k+1) n+1 \leq T 1(n) \leq(k+2) n+1 .
$$


Better performance can be obtained by using two systolic arrays as shown in Figure 8. In this new arrangement both array can be fed in simultaneously by two row tiles improving thus the performance. If one tile is larger than the other, once the shorter one ends, a new tile could feed the idle systolic array. This way we can have more than two tiles traveling through the systolic array. Using the proposed systolic array we divide $n n z$ by 2 and each half will enter a different linear array. The time taken by the new systolic array is presented as $T 2(n)$ in Table 1.

$$
\left\lceil\frac{n n z}{2}\right\rceil+n+2 \leq T 2(n) \leq\left\lceil\frac{n n z}{2}\right\rceil+2 n+2 .
$$

As can be observed both algorithms are $\mathrm{O}(\mathrm{n})$ because their running time grows linearly depending on the input size.

Table 1: Table The basic characteristic of each matrix used in our time analysis.

\begin{tabular}{|c|c|c|c|c|c|c|}
\hline & $\begin{array}{c}\text { Name } \\
\text { (Application Area) }\end{array}$ & Dimension & NNZ & $\begin{array}{c}\text { Nun tjd } \\
\text { (row_tiles) }\end{array}$ & $\begin{array}{c}\text { T1(n) } \\
\text { steps }\end{array}$ & $\begin{array}{c}\text { T2(n) } \\
\text { steps }\end{array}$ \\
\hline 1 & $\begin{array}{c}\text { LNS131 } \\
\text { (Fluid flow modeling) }\end{array}$ & $131 \times 131$ & 536 & 13 & 799 & $\approx 520$ \\
\hline 2 & $\begin{array}{c}\text { BP200 } \\
\text { (Simplex method basis matrix) }\end{array}$ & $822 \times 822$ & 3802 & 21 & 5447 & $\approx 3546$ \\
\hline 3 & $\begin{array}{c}\text { CURTIS54 } \\
\text { (Stiff Biochemical ODEs) }\end{array}$ & $54 \times 54$ & 291 & 16 & 400 & $\approx 255$ \\
\hline 5 & $\begin{array}{c}\text { GRE115 } \\
\text { (Simulation of computer systems) }\end{array}$ & $115 \times 115$ & 421 & 7 & 652 & $\approx 444$ \\
\hline 6 & $\begin{array}{c}\text { MEMPLUS } \\
\text { (Circuit design) }\end{array}$ & $17,758 \times 17,758$ & 126.150 & 574 & 161,667 & $\approx 98,592$ \\
\hline $\begin{array}{c}\text { PORES } \\
\text { (Reservoir modeling) }\end{array}$ & $30 \times 30$ & 180 & 10 & 241 & $\approx 151$ \\
\hline
\end{tabular}

Although we have presented a systolic array where matrices have been compressed using TJDS and the tiles selected by rows, a combination of column tiles and row tiles can be used to feed in the systolic array. This approach might reduce the number of calls made to the systolic array. Figure 9 presents three different organizations of Matrix CURTIS54. The one on top shows the matrix compressed using TJDS and divided in half to feed in the systolic array shown in Figure 8. Each linear works with 50\% of the matrix coefficients. The one in the middle presents the matrix coefficients compressed with TJDS but ready to enter the array using a combination of row tile and column tiles. The one at the bottom shows the matrix compressed with CSC. using this approach all matrix coefficients could enter the two arrays columnwise or, if you observe carefully, adapting tiling is also supported by combining column tiles and row tiles of diferrent sizes (Kung, McDanel, and Zhang 2018). In this arrangement you can see that the three top row tiles are dense. The disadvantage of using solely CCS is that more calls must be made to the systolic array. If you take a look to Figure 9 you will observe that in matrix CURTIS54 there are 54 column tiles and 16 row tiles (tj_tiles). Nevertheless, CCS is very effective to reduce the number of row tiles by pruning column tiles. For instance, observing the middle data arrangement in Figure 9, if we can call the systolic array to handle the left hand side column first (largest column), then the number of row tiles is reduced from 16 to 10. This will speed up the computation because the systolic array will be called 11 times instead the 16 calls required in the original arrangement. A more significant example could be observed when handling matrix soc-signbitcoin-otc, which generates 535 tiles. In this matrix the first four more populated column-tiles have 535, 412, 311, and 279 coefficients respectively. By pruning the first three columns the number of row-tiles is 
reduced to 279 and this means a reduction of 52 percent in the number of row-tiles to be handled by the systolic array.


Figure 9: Matrix Curtis54 organized in column and row tiles.

\section{FINAL REMARKS}

We have presented a systolic array with $2 n+2$ processing elements to compute the sparse matrix vector product in $T 2(n) \approx\left\lceil\frac{n n z}{2}\right\rceil+2 n+2$. Although we have presented the matrix compressed using TJDS this systolic array can handle matrices compressed with CSC as well or a combination of both. More linear systolic arrays can be added but we think that more than four will be a waste of resources compare to the gain in performance. As the length of the systolic arrays grows depending on $n$, we envision a systolic array similar to the one shown in Figure 8 but with four rows; each row consisting of a multiplier and 64 selective adders. This arrangement will allow to handle up to four tiles in parallel. To handle large matrices, partitioning by rows is recommended to compute the resulting vector $y$. By selecting partitions of 64 rows, sixty four elements of the resulting vector $y$ can be obtained on each call to the systolic array.

\section{ACKNOWLEDGMENTS}

We would like thank the anonymous referees for their review, which helped improve the paper. We also thank Archita Kale who participated in discussions for the column-tile strategy.

\section{REFERENCES}

Boisvert, R., R. Pozo, K. Remington, R. F. Barrett, and J. J. Dongarra. 1997. "Matrix Market: a web resource for test matrix collections". In Proceedings of the IFIP TC2/WG2.5 Working Conference on the Quality of Numerical Software, Assessment and Enhancement, pp. 125-137. London, Chapman and Hall.

Davis, T. A., and Y. Hu. 2011. "The University of Florida Sparse Matrix Collection". ACM Trans. Math. Softw. vol. 38 (1), pp. 1-25. 
Dorrance, R., F. Ren, and D. Marković. 2014. "A scalable sparse matrix-vector multiplication kernel for energy-efficient sparse-blas on FPGAs". In FPGA '14 Proceedings of the 2014 ACM/SIGDA international symposium on Field-programmable gate arrays, pp. 161-170.

Han, S., H. Mao, J. Pu, A. Pedram, M. A. Horowitz, and W. J. Dally. 2016. "EIE: Efficient Inference Engine on Compressed Deep Neural Network". In Proceedings of the 2016 ACM/IEEE 43rd Annual International Symposium on Computer Architecture, pp. 243-254.

Im, E. J., K. Yelick, and R. Vuduc. 2004. "SPARSITY: An Optimization Framework for Sparse Matrix Kernels". International Journal of High Performance Computing Applications vol. 18 (1), pp. 135-158.

Jouppi, N. P., C. Young, N. Patil, D. Patterson, G. Agrawal, R. Bajwa, S. Bates, S. Bhatia, N. Boden, and A. Borchers. 2017. "In-datacenter performance analysis of a tensor processing unit". In Proceedings of the 44th Annual International Symposium on Computer Architecture. ACM, pp. 1-12.

Krotkiewski, M., and M. Dabrowski. 2010. "Parallel symmetric sparse matrix-vector product on scalar multi-core CPUs". Parallel Computing vol. 36 (4), pp. 181-198.

Kung, H. T. 1982. "Why Systolic Architectures?”. Computer vol. 15 (3), pp. 37-46.

Kung, H. T., and C. E. Leiserson. 1979. "Systolic arrays (for vlsi)". In Sparse Matrix Proceedings 1978, pp. 256-282, Society for Industrial and Applied Mathematics.

Kung, H. T., B. McDanel, and S. Q. Zhang. 2018. "Adaptive Tiling: Applying Fixed-size Systolic Arrays To Sparse Convolutional Neural Networks". In 24th International Conference on Pattern Recognition (ICPR 2018), pp. 1006-1011.

Montagne, E., and A. Ekambaran. 2004. "An Optimal Storage Format for Sparse Matrices". Information Processing Letters vol. 90 (2), pp. 87-92.

Vuduc, R. W. January 2004. Automatic Performance Tuning of Sparse Matrix Kernels. Ph.D. thesis, University of California, Berkeley, CA, USA.

Williams, S., L. Oliker, R. Vuduc, J. Shalf, K. Yelick, and J. Demmel. 2009. "Optimization of sparse matrixvector multiplication on emerging multicore platforms". Parallel Computing vol. 35 (3), pp. 178-194.

Zhuo, L., and V. K. Prasanna. 2005. "Sparse Matrix-Vector Multiplication on FPGAs". In FPGA '05 Proceedings of the 2005 ACM/SIGDA 13th international symposium on Field-programmable gate arrays, pp. 63-74.

\section{AUTHOR BIOGRAPHIES}

EURIPIDES MONTAGNE is an Associate Lecturer in the Department of Computer Science at the University of Central Florida. He holds a Ph.D. from Universidad Central de Venezuela. His research interests include program optimization, computer architecture and irregular problems. His email address is eurip@eecs.ucf.edu.

RINA SUROS is a Professor in the Department of Computer Science at the Universidad Central de Venezuela. She holds a Ph.D. in Computer Science from Université Paris-Sud (Orsay). Her research interests include high performance computing, and big data. Her email address is rsuros@ gmail.com. 